\title{
Jet fragmentation as a tool to explore double parton scattering using Z-boson + jets processes at the LHC
}

\author{
R. Kumar \\ Akal University, Talwandi Sabo 151302, India \\ M. Bansal \\ D.A.V. College, Sector 10, Chandigarh 160010, India \\ S. Bansal ${ }^{\dagger}$ \\ Panjab University, Chandigarh 160014, India \\ (Received 10 August 2018; published 17 May 2019)
}

\begin{abstract}
The Large Hadron Collider witnesses the highest ever production cross section of double parton scattering processes. The production of a Z-boson along with two jets from double parton scattering provides a unique opportunity to explore the kinematics of double parton scattering processes and their dependence on the scale of the second interaction. The experimental measurement of this process is largely contaminated by $Z+$ jets production from single parton scattering. In this paper, fragmentation properties of a jet are explored to check their sensitivity toward double parton scattering. The present study is performed using simulated $\mathrm{Z}+$ jets events, produced with MADGRAPH and POWHEG Monte-Carlo event generators, hadronized and parton showered using PYTHIA8. The effect of different hadronization models on the discrimination based on the fragmentation properties of a jet is also investigated by using events simulated with HERWIG++. It is observed that discrimination based on the fragmentation properties of a jet can significantly suppress the background from single parton scattering, which results into 40\%-50\% gain in the contribution of double parton scattering.
\end{abstract}

DOI: 10.1103/PhysRevD.99.094025

\section{INTRODUCTION}

The large collision energy and involved parton densities in a proton-proton (pp) collision at Large Hadron Collider (LHC) lead to significant increase in probability of more than one parton-parton scattering in the same pp collision. The additional scatterings, along with primary hard partonparton scattering, are called multiple parton interactions (MPI) [1]. Usually, MPI produce particles with relatively small transverse momenta $\left(p_{\mathrm{T}}\right)$, but there is a possibility of producing particles with large $p_{\mathrm{T}}$ or mass, such as jets and/ or vector (W/Z) bosons. The production of such particles from at least two parton-parton scatterings is referred to as double parton scattering (DPS). The study of DPS provides vital information on the parton-parton correlations and parton distributions in a hadron [2]. In addition, DPS also

\footnotetext{
*raman_phy@auts.ac.in

†Sunil.Bansal@cern.ch
}

Published by the American Physical Society under the terms of the Creative Commons Attribution 4.0 International license. Further distribution of this work must maintain attribution to the author(s) and the published article's title, journal citation, and DOI. Funded by SCOAP. constitutes as a background to new physics searches [3,4]. A broad range of measurements is available for DPS processes at different collision energies, performed using different final states, e.g., $W / Z+$ jets, photon + jets, 4-jets, diboson processes [5-14], etc.

The production of $Z+2$-jets from DPS is important because of large production cross section and it also provides the opportunity to study the dependence on the scale of the second hard interaction, as predicted by theoretical quantum chromodynamics (QCD) models $[15,16]$. The experimental measurements of these DPS processes are dominated by production of $Z+$ jets from single parton scattering (SPS). In order to increase DPS sensitivity and study its kinematic properties, it is essential to devise methods to control SPS background with minimal effect on the DPS. In the existing experimental as well as theoretical studies, DPS and SPS processes are distinguished using the kinematical and correlation properties of Z-boson and jets [10,11,17-19]. This paper presents the studies, using $Z+$ jets process, to demonstrate that fragmentation properties of a jet can be effective in suppressing the SPS background.

The outline of the paper is as follows. The simulation of the $Z+$ jets events and selection criteria is discussed in 
Sec. II. In Sec. III, fragmentation properties of a jet and related variables are discussed. The results of the present study are discussed in the Sec. IV and finally Sec. V summarizes the paper.

\section{EVENT GENERATION AND SELECTION CRITERIA}

\section{A. Event generation}

The present study requires events simulated with MonteCarlo event generators, which are able to produce sufficient number of jets in association with Z-boson. The event generators MADGRAPH [20,21] and POWHEG [22,23] are used to simulate $Z+$ jets events in the present study. MADGRAPH is a tree-level matrix element event generator, which is able to produce up to 4 jets as per matrix element leading order (LO) calculations. The distributions of $\mathrm{Z}+$ jets, produced by LHC, are well described by MADGRAPH [24]. POWHEG produces $Z+2$-jets events up to next-to-leading order (NLO) using "multiscale improved NLO" (MiNLO) method [23,25], which is also capable to describe well the jets production associated with Z-boson. These events are further hadronized and parton showered with PYTHIA8 [26]. MPI are included while parton showering with PYTHIA8 [27].

The production of $Z+2$-jets events from DPS processes is simulated with PYTHIA8 event generator, which is configurable to specify process involved and interaction scale independently for the two parton-parton scatterings. In the present analysis, first parton-parton scattering is configured to produce Z-boson, whereas two jets are produced from the second parton-parton scattering.

All these processes are produced in $\mathrm{pp}$ collisions assuming a center-of-mass energy of $13 \mathrm{TeV}$ and using ATLAS A14 tune [28] with NNPDF2.3LO set of parton distribution functions (PDF) for the simulation of MPI model. This version of MPI tune is derived by fitting the underlying event data collected at the LHC.

Dijet QCD events are simulated using PYTHIA8 with A14 tune. These processes are also simulated using HERWIG++ (version 2.7.0) [29] and CUETHppS1 tune [30].

\section{B. Event selection}

In these simulated events, Z-boson candidates are identified using four-momenta of the muons coming from the decay of Z-boson. An event is considered to have a Z-candidate if it satisfies the following conditions:

(i) presence of two muons with $p_{\mathrm{T}}$ larger than $20 \mathrm{GeV} / c$ and absolute pseudorapidity $(\eta)$ less than 2.5 .

(ii) Invariant mass of the two selected muons is required to be in range of $60-120 \mathrm{GeV} / c^{2}$.

This selection criteria is motivated from the trigger and the background constraints for the measurements involving Z-boson at the LHC experiments. These events, having a $\mathrm{Z}$ candidate, are required to have at least two jets with a minimal $p_{\mathrm{T}}$ of $20 \mathrm{GeV} / c$ and $|\eta|<2.5$. The jets are clustered using the anti- $k_{T}$ algorithm [31] with the radius parameter equal to 0.5 using the FASTJET software package [32].

\section{METHODOLOGY}

The production of $Z+$ jets events from DPS includes 2 -jets production from the second interaction, which is dominated with the jets initiated by gluons. Whereas, the jets produced in association with Z-boson via SPS are expected to be predominately initiated by quarks. Figure 1 shows the fraction of gluon-initiated jets, in $Z+$ jets events produced via DPS and SPS, as a function of the jet $p_{\mathrm{T}}$. A jet is tagged as gluon-initiated or quark-initiated by matching the jet with partons in $\eta \times \phi$ space. A distance parameter $\Delta \mathrm{R}$ is defined as:

$$
\Delta R=\sqrt{\left(\eta_{\text {jet }}-\eta_{\text {parton }}\right)^{2}+\left(\phi_{\text {jet }}-\phi_{\text {parton }}\right)^{2}} .
$$

A pair of jet-parton is considered to be matched if $\Delta \mathrm{R}$ is smaller than 0.3 . It is observed that $\approx 75 \%$ of jets in the DPS sample are gluon-initiated whereas in SPS sample the fraction of gluon-initiated jets is relatively small $(\approx 45 \%)$. Thus, contribution of DPS events can be increased if flavor of a jet can be identified and only events with gluoninitiated jets are considered.

It is well established that the fragmentation properties of the quarks and the gluons are different from each other [33-39]. In particular, the jets initiated by the gluons show significantly different behavior with respect to those

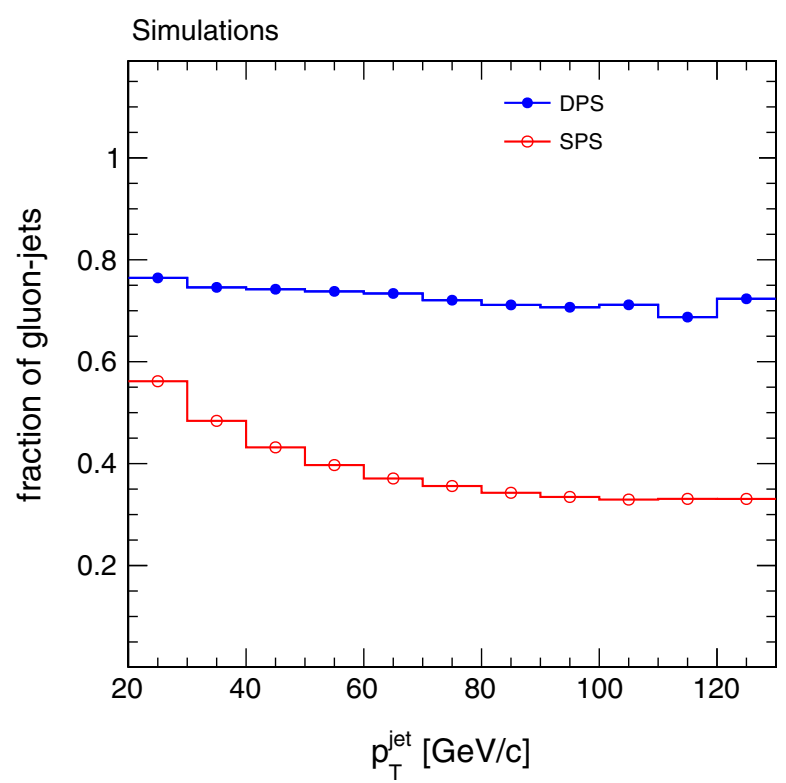

FIG. 1. The fraction of gluon-initiated jets as a function of the jet $p_{\mathrm{T}}$ in the simulated DPS (blue solid circle markers) and SPS (red hollow circle markers) events. 
initiated by light-flavor quarks (u, d, s). There are number of observables [37-40] which can be constructed using intrinsic properties of a jet. The study presented in this article is aimed to emphasize on use of intrinsic properties of jets for SPS background suppression rather than to construct the best quark-gluon discriminator. Therefore, only a certain number of observables are considered in the analysis as discussed below:

(i) Jet size $\left(\sigma^{\text {jet }}\right)$ : A jet, with conical structure, is approximated by an ellipse when projected in $\eta \times \phi$ space. Two principal axes of the ellipse are used which are represented as major axis $\left(\sigma_{1}^{\text {jet }}\right)$ and minor axis $\left(\sigma_{2}^{\text {jet }}\right)$ of the jet cone.

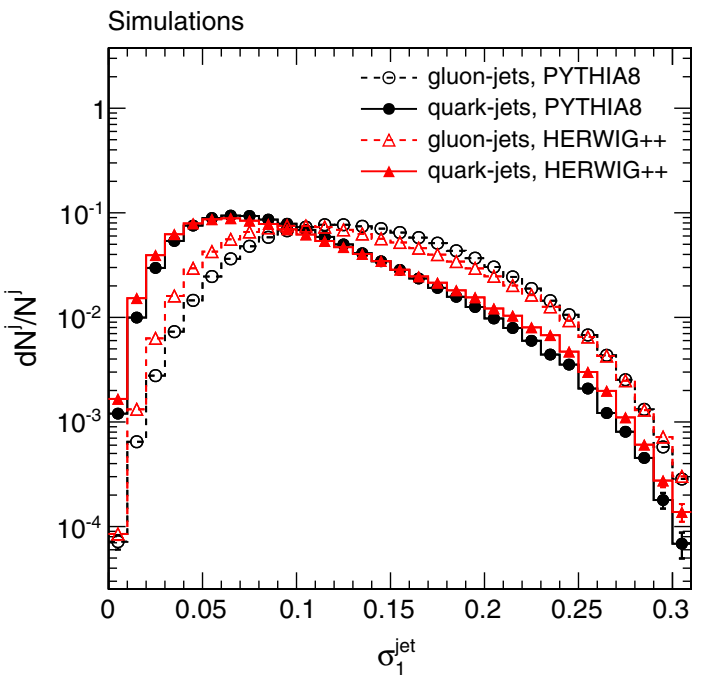

(a)

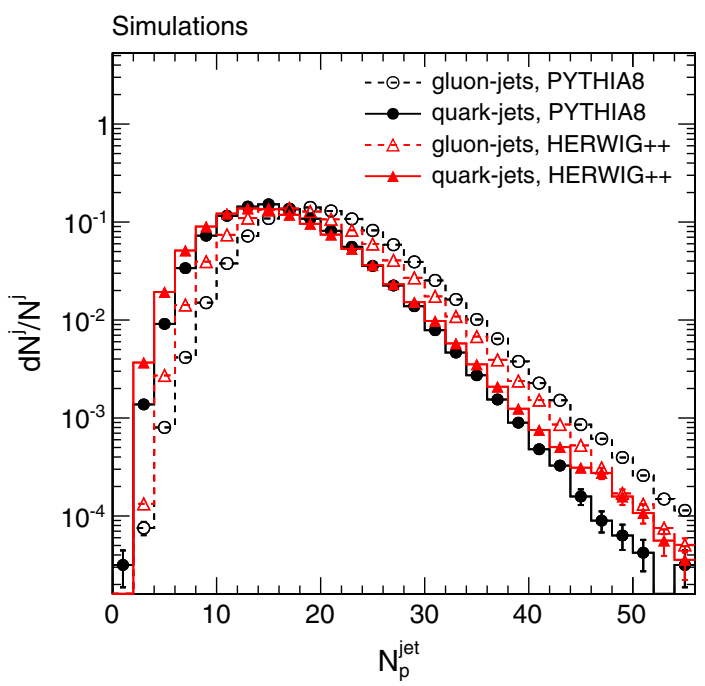

(c) (ii) Jet constituents multiplicity $\left(N_{p}^{\mathrm{jet}}\right)$, which is the total number of particles (charged or neutral) within the jet.

(iii) Jet fragmentation function $\left(p_{\mathrm{T}}^{\mathrm{jet}} D\right)$, which represents the $p_{\mathrm{T}}$-distribution among the constituents of a jet. It is defined as:

$$
p_{\mathrm{T}}^{\text {jet }} D=\frac{\sqrt{\sum_{i} p_{\mathrm{T}, \mathrm{i}}^{2}}}{\sum_{i} p_{\mathrm{T}, \mathrm{i}}},
$$

where sum extends over the jet constituents and $p_{\mathrm{T}, \mathrm{i}}$ represents the $p_{\mathrm{T}}$ of $i$ th constituent. The value of

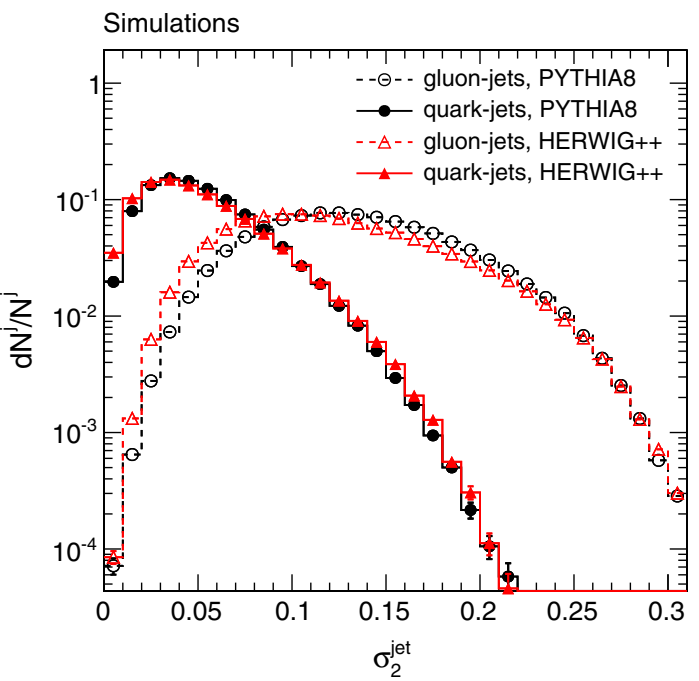

(b)

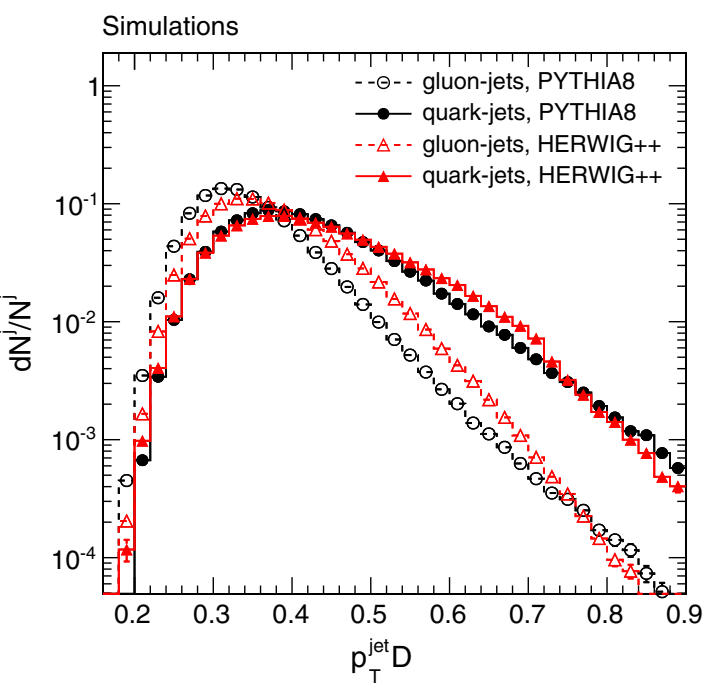

(d)

FIG. 2. The distributions of discriminating variables: (a) major axis size of jet cone $\left(\sigma_{1}^{\text {jet }}\right)$, (b) minor axis size of jet cone $\left(\sigma_{2}^{\text {jet }}\right)$, (c) jet constituents multiplicity $\left(N_{p}^{\mathrm{jet}}\right)$, and (d) jet fragmentation function $\left(p_{\mathrm{T}}^{\text {jet }} D\right)$ are compared for gluon-initiated jets (hollow markers) and quark-initiated jets (solid markers) events hadronized and parton showered with PYTHIA8 (black colored markers) and HERWIG++ (with red colored markers). 
$p_{\mathrm{T}}^{\mathrm{jet}} D$ for a jet will be equal to one if there is only one constituent for that particular jet. The jets with infinite number of jet constituents will have value of $p_{\mathrm{T}}^{\text {jet }} D$ approaching towards zero.

More details of these observables can be found in Refs. [39,40]. The distributions of these discriminating variables for gluon-initiated jets and quark-initiated jets are shown in Fig. 2. These distributions are constructed using dijet events simulated with PYTHIA8 and as well as using HERWIG++. The gluon-initiated and quark-initiated jets are identified with jet-parton matching in $\eta \times \phi$ space as described earlier. It is clear from these distributions that gluon-initiated jets are broader than quark-initiated jets and have larger number of particles. The constituents of the quark-initiated jets are hard as compared to the constituents of the gluon-initiated jets. Therefore, the quark-initiated jets have a harder fragmentation function as compared to gluoninitiated ones. Thus, for these observables a clear distinction between gluon- and quark-initiated jets is visible. The effectiveness of the usage of these variables is investigated with cut-based and multivariate analysis (MVA) methods.

\section{RESULTS AND DISCUSSIONS}

In $Z+$ jets events simulated with MADGRAPH + PYTHIA8, two jets are required along with a Z-boson as per the kinematic selection criteria mentioned in Sec. II. The selected $Z+2$-jets events contains the contribution from DPS as well as SPS processes. A selected event is considered to be produced by DPS, if there are two MPI partons present within the acceptance, otherwise event is considered as SPS background [10]. The fraction of DPS processes contributing in selected $Z+2$-jets sample is about 0.075 which is consistent with the previous studies [10]. The effect of the observables mentioned in Sec. III, which are sensitive to quark-gluon discrimination, is evaluated by calculating the gain in DPS fraction after requiring selected two jets to be gluon-initiated.

In cut-based method, the jets are considered to be gluoninitiated if two selected jets satisfy the conditions listed in Table I. These cuts are optimized by maximizing the figure of merit defined as $S / \sqrt{S+B}$, where $S$ represents gluoninitiated jets and $B$ represents quark-initiated jets. This set of selection criteria selects $\approx 82 \%$ of gluon-initiated jets with $54 \%$ rejection of quark-initiated jets. When both of the

TABLE I. Conditions on observables for selection of gluoninitiated jets in cut-based analysis.

\begin{tabular}{lc}
\hline \hline Observable & Condition \\
\hline$\sigma_{1}^{\text {jet }}$ & $>0.04$ \\
$\sigma_{2}^{\text {jet }}$ & $>0.02$ \\
$N_{p}^{\text {jet }}$ & $>12.0$ \\
$p_{\mathrm{T}}^{\text {jet }} D$ & $<0.49$ \\
\hline \hline
\end{tabular}

jets are required to be gluon initiated, DPS fraction comes out to be 0.106 which is $41 \%$ larger as compared to selection when fragmentation properties of the jets were not used.

To further enhance the sensitivity and to consider the possible correlations between observables, MVA method is used. This method is based on boosted decision trees (BDT) implemented in the TMVA framework [41]. The discriminating observables along with the $p_{\mathrm{T}}$ and $\eta$ of jets are provided as an input to the BDT. For MVA training, these observables are constructed using dijet events simulated with PYTHIA8. To minimize the statistical bias, independent event samples of gluon- and quark-initiated jets are used for the training and testing of the MVA. The distribution of the BDT discriminant for gluon- and quarkinitiated jets is shown in Fig. 3, which gives a clear distinction between two flavors of jets. The MVA output is used to tag a jet either as quark-initiated or gluoninitiated. The selected jets are tagged as gluon-initiated jets with requirement of BDT value greater than -0.105 , otherwise considered as initiated by quarks. This criteria selects $\approx 90 \%$ of gluon-initiated jets with $50 \%$ rejection of quark-initiated jets. This trained MVA is used to select gluon-initiated jets in the $Z+2$-jets events. If both jets in the selected $Z+2$-jets events are required to be initiated by gluons, SPS contribution got reduced by $48 \%$ while keeping $72 \%$ of the DPS events. In other words, DPS fraction in $Z+2$-jets sample is now about 0.113 which is $20 \%$ larger as compared to the cut-based analysis.

The presence of MPI adds soft particles which can affect the intrinsic properties of a jet. It is observed that for the same quark-jet efficiency, the selection efficiency for

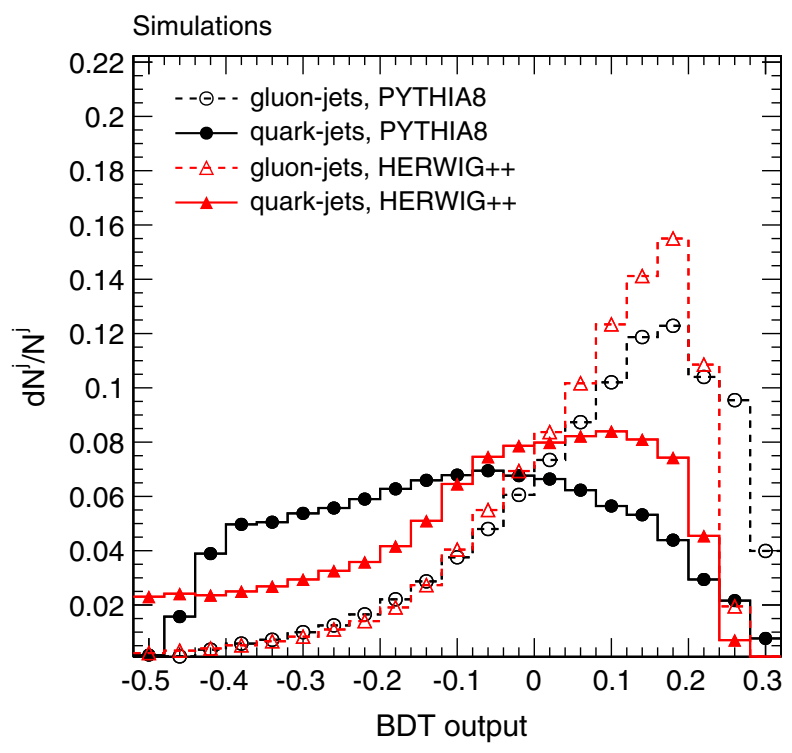

FIG. 3. BDT output for gluon-initiated (hollow markers) jets and quark-initiated jets (solid markers) in case of dijet events produced with PYTHIA8 (black colored markers) and HERWIG++ (red colored markers). 


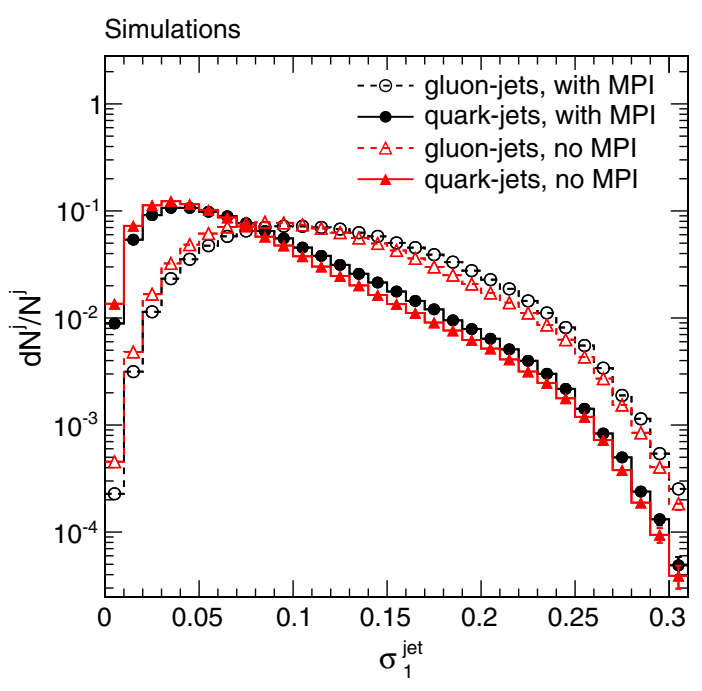

(a)

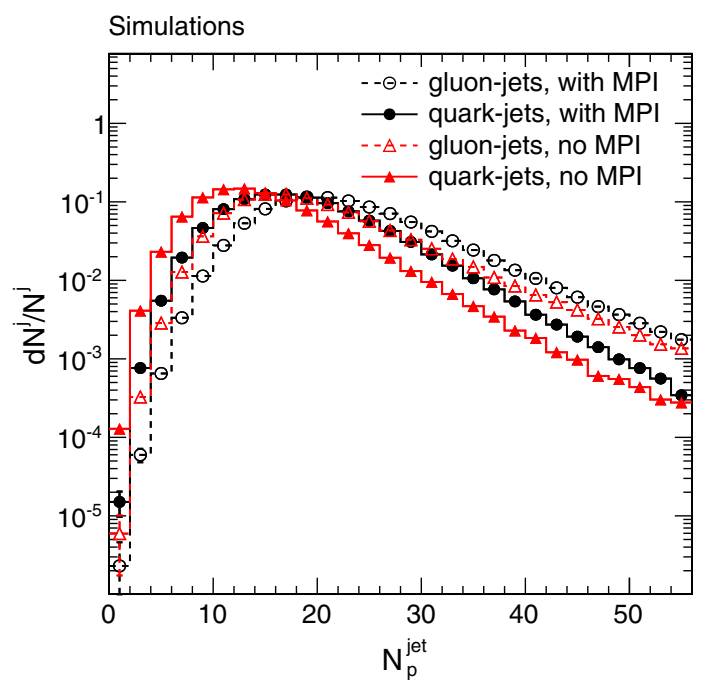

(c)

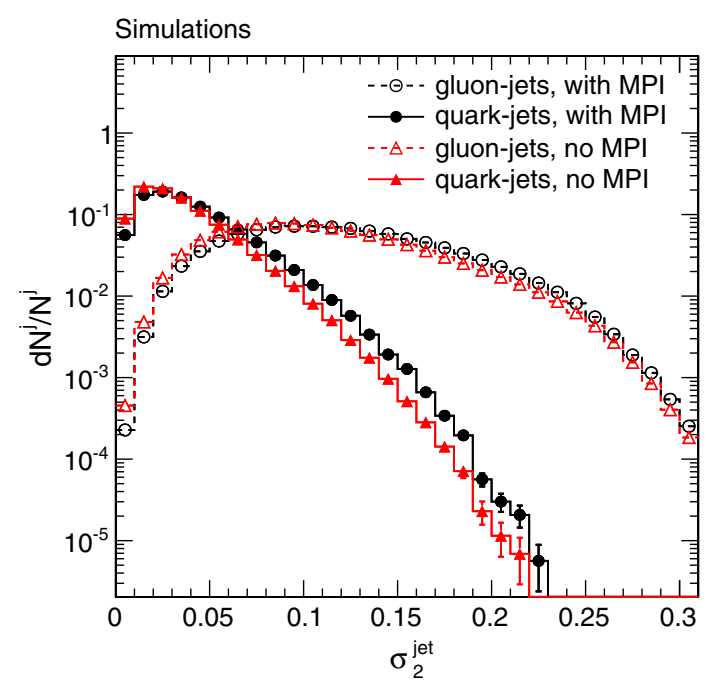

(b)

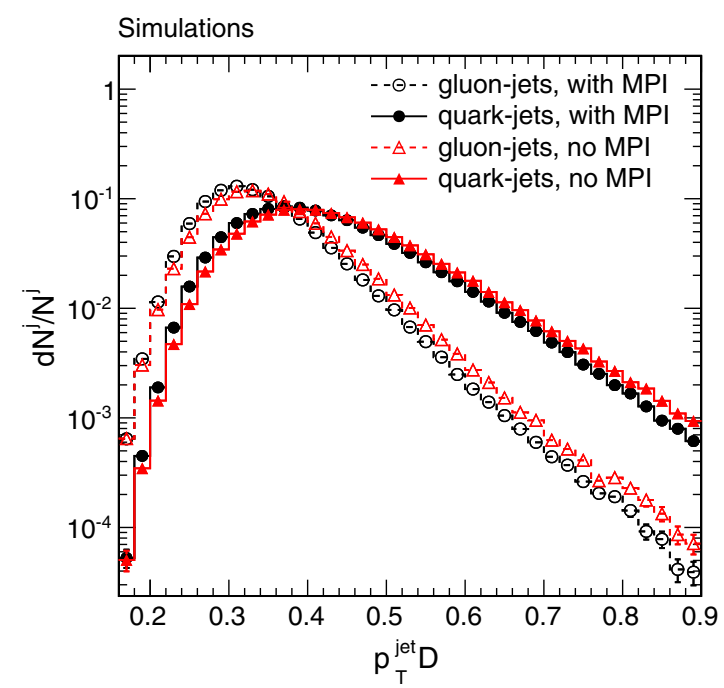

(d)

FIG. 4. The distributions of discriminating variables: (a) major axis size of jet cone $\left(\sigma_{1}^{\text {jet }}\right)$, (b) minor axis size of jet cone $\left(\sigma_{2}^{\text {jet}}\right)$, (c) jet constituents multiplicity $\left(N_{p}^{\text {jet }}\right)$, and (d) jet fragmentation function $\left(p_{\mathrm{T}}^{\text {jet }} D\right)$ are compared for gluon-initiated jets (hollow markers) and quark-initiated jets (solid markers) $Z+2$-jets events generated with MADGRAPH + PYTHIA8 with MPI (black colored markers) and without MPI (red colored markers).

gluon-initiated jets is about $92 \%$ if MPI is switched off during event generation. The effect of MPI on the input variables is shown in Fig. 4. Different hadronization models can also affect the discrimination based on fragmentation properties of a jet. As shown in Fig. 2, there are significant differences in shape of input variables for PYTHIA8 and HERWIG++. To investigate the possible effects of different hadronization models on the DPS fraction, the BDT discriminator is trained using events parton showered and hadronized with HERWIG++. It is observed that for same efficiency of quark-initiated jets, the selection efficiency for gluon-initiated jets is $15 \%$ lower if HERWIG++ is used in place of PYTHIA8 for the training of BDT. This difference in hadronization properties results in reduction of DPS fraction to 0.107 , but still there is a gain of $43 \%$ as compared to selection when fragmentation properties of the jets were not used.

To further corroborate effectiveness of the method, the $Z+$ jets events are generated using POWHEG, which are hadronized and parton showered using PYTHIA8. With this simulation, there is a similar rejection of SPS background, which results in $36 \%$ gain in the DPS fraction for inclusive $Z+2$-jets sample. The differences in the SPS rejection for POWHEG and MADGRAPH is expected due to the differences in treatment of the LO and NLO effects which also change relative fraction of quark- and gluon-initiated jets.

There are studies which show that SPS background can be suppressed by restricting the boost of Z-boson [42]. 
The SPS background is suppressed with an upper cut on dilepton $p_{\mathrm{T}}$ less than $10 \mathrm{GeV} / c$ with DPS fraction of 0.32 in the selected events $Z+2$-jets events. The effect of jet-tagging is checked on top of the cut on the boost of Z-boson. Using jet fragmentation properties, in addition to Z-boost cut, as discriminator DPS fraction comes out to be 0.42 . Now, gain in DPS fraction with jet fragmentation is relatively smaller as compared to no condition on the dilepton $p_{\mathrm{T}}$. This behavior is expected because after restricting the boost of Z-boson, most of the remaining jets will be coming from initial- or final-state radiation and hence, will be dominated by gluons.

The quark-gluon discriminator can be optimized for better discrimination between gluon- and quark-initiated jets with consideration of additional infrared and collinear (IRC) safe observables [37-40]. To study the effect of inclusion of IRC safe observables on the results, jet mass, jet shape and girth are considered in addition to the observables mentioned in Sec. III to construct the discriminator. Qualitatively, the suppression of SPS background is still there and DPS fraction in selected $Z+$ jets events increases to 0.119 , which is equivalent to a gain of $59 \%$ as compared to selection when fragmentation properties of the jets were not used.

It is conclusive from these studies that intrinsic properties of jets can be used to enhance the sensitivity to DPS, where two jets are produced from second interaction. The different hadronization models have varying discrimination which can be considered as a part of the systematic uncertainty during the measurements. The jets initiated by heavy quarks (in particular, b-quarks) show fragmentation properties similar to gluon-initiated jets. Therefore, it is required to veto these jets for effective separation between quark- and gluon-tagged jets. These jets, from b-quarks, can be efficiently identified with the use of available b-tagging algorithms $[43,44]$. The acceptance and pileup conditions in the LHC experiments can affect the above sensitivity and need to be studied with the actual experimental environment.

\section{SUMMARY}

This paper presents a feasibility study of the $Z+$ jets events to explore the possibility to enhance DPS sensitivity by suppressing SPS background with the use of differences in the fragmentation properties of jets initiated by quarks and gluons. The $Z+$ jets events are simulated with MADGRAPH and POWHEG Monte-Carlo event generators, followed by hadronization and showering with PYTHIA8. A number of observables, defining the fragmentation behavior of a jet, are used as input for the multivariate analysis and subsequently used to tag a jet as gluon-initiated or quark-initiated. It is observed that with the discrimination of gluon- and quark-initiated jet, it is possible to suppress SPS background up to $52 \%$ resulting in a $40 \%-50 \%$ gain in $Z+2$-jets events produced by DPS. The effect of different hadronization model on the discrimination is also investigated by using events simulated with HERWIG++. As expected, HERWIG++ gives different sensitivity but SPS suppression is still there. It will be interesting to study the impact of these variables on the DPS measurements in actual experimental conditions, i.e., pileup, detector acceptance, at the LHC.

\section{ACKNOWLEDGMENTS}

This work is financially supported by Department of Science and Technology (DST), New Delhi and University Grants Commission (UGC), New Delhi.
[1] T. Sjöstrand and M. Van Zijl, Phys. Rev. D 36, 2019 (1987).

[2] M. Diehl, D. Ostermeier, and A. Schäfer, J. High Energy Phys. 03 (2012) 89; 03 (2016) 001.

[3] M. Y. Hussein, Nucl. Phys. B, Proc. Suppl. 174, 55 (2007).

[4] S. Chatrchyan et al. (CMS Collaboration), J. High Energy Phys. 06 (2011) 077.

[5] J. Alitti et al. (UA2 Collaboration), Phys. Lett. B 268, 145 (1991).

[6] T. Akesson et al. (AFS Collaboration), Z. Phys. C 34, 163 (1987).

[7] V. M. Abazov et al. (D0 Collaboration), Phys. Rev. D 81, 052012 (2010).

[8] F. Abe et al. (CDF Collaboration), Phys. Rev. D 56, 3811 (1997).
[9] F. Abe et al. (CDF Collaboration), Phys. Rev. D 47, 4857 (1993).

[10] S. Chatrchyan et al. (CMS Collaboration), J. High Energy Phys. 03 (2014) 032.

[11] G. Aad et al. (ATLAS Collaboration), New J. Phys. 15, 033038 (2013).

[12] Chatrchyan et al. (CMS Collaboration), CERN Report No. CMS PAS-FSQ-12-017, 2015, https://cds.cern.ch/ record/2007815? $\ln =\mathrm{en}$.

[13] A. M. Sirunyan et al. (CMS Collaboration), J. High Energy Phys. 02 (2018) 032.

[14] M. Aaboud et al. (ATLAS Collaboration), J. High Energy Phys. 11 (2016) 110.

[15] B. Blok and M. Strikman, Adv. Ser. Dir. High Energy Phys. 29, 63 (2018). 
[16] A. M. Snigirev, Phys. Rev. D 81, 065014 (2010).

[17] B. Blok and P. Gunnellini, Eur. Phys. J. C 76, 202 (2016).

[18] E. Maina, J. High Energy Phys. 01 (2011) 061.

[19] Q.-H. Cao, Y. Liu, K.-P. Xie, and B. Yan, Phys. Rev. D 97, 035013 (2018).

[20] J. Alwall, M. Herquet, F. Maltoni, O. Mattelaer, and T. Stelzer, J. High Energy Phys. 06 (2011) 128.

[21] F. Maltoni and T. Stelzer, J. High Energy Phys. 02 (2003) 027.

[22] S. Frixione, P. Nason, and C. Oleari, J. High Energy Phys. 11 (2007) 070.

[23] J. M. Campbell, R. K. Ellis, P. Nason, and G. Zanderighi, J. High Energy Phys. 08 (2013) 005.

[24] V. Khachatryan et al. (CMS Collaboration), J. High Energy Phys. 04 (2017) 022.

[25] K. Hamilton, P. Nason, and G. Zanderighi, J. High Energy Phys. 10 (2012) 155.

[26] T. Sjöstrand, S. Mrenna, and P. Z. Skands, Comput. Phys. Commun. 178, 852 (2008).

[27] R. Corke, arXiv:0901.2852.

[28] G. Aad et al. (ATLAS Collaboration), CERN Report No. ATL-PHYS-PUB-2012-003, 2012.

[29] M. Bähr et al., Eur. Phys. J. C 58, 639 (2008).

[30] V. Khachatryan et al. (CMS Collaboration), Eur. Phys. J. C 76, 155 (2016).

[31] M. Cacciari, G. P. Salam, and G. Soyez, J. High Energy Phys. 04 (2008) 063.
[32] M. Cacciari, G. P. Salam, and G. Soyez, Eur. Phys. J. C 72, 1896 (2012).

[33] OPAL Collaboration, Z. Phys. C 58, 387 (1993).

[34] R. Akers et al. (OPAL Collaboration), Z. Phys. C 68, 179 (1995).

[35] P. Abreu et al. (DELPHI Collaboration), Z. Phys. C 70, 179 (1996).

[36] D. Buskulic et al. (ALEPH Collaboration), Phys. Lett. B 384, 353 (1996).

[37] J. Gallicchio and M. D. Schwartz, Phys. Rev. Lett. 107, 172001 (2011).

[38] G. Aad et al. (ATLAS Collaboration), Eur. Phys. J. C 74, 3023 (2014).

[39] Chatrchyan et al. (CMS Collaboration), CERN Report No. CMS PAS-JME-13-002, 2013, https://cds.cern.ch/ record $/ 1599732 ? \ln =$ en.

[40] T. Cornelis (CMS Collaboration), SLAC, Report No. SLACeconf-C140602.2, 2014, https://cds.cern.ch/record/1754897/.

[41] A. Hoecker, P. Speckmayer, J. Stelzer, J. Therhaag, E. von Toerne, and H. Voss, Proc. Sci., ACAT2007 (2007) 040 [arXiv:physics/0703039].

[42] R. Kumar, M. Bansal, S. Bansal, and J. B. Singh, Phys. Rev. D 93, 054019 (2016).

[43] G. Aad et al. (ATLAS Collaboration), J. Instrum. 11, P04008 (2016).

[44] Chatrchyan et al. (CMS Collaboration), Report No. CMS-PAS-BTV-15-001, 2016, https://cds.cern.ch/ record/2138504. 